\title{
Hadron production measurements from NA61/SHINE for LBL neutrino experiments
}

\author{
Alexander Korzenev*, on behalf of the NA61/SHINE collaboration \\ DPNC, University of Geneva, Quai Ansermet 24, CH-1205 Geneva, Switzerland \\ E-mail: Alexanter.Korzenevecern.ch
}

\begin{abstract}
Modern accelerator neutrino experiments require a precise knowledge of initial neutrino fluxes. In many cases an uncertainty of this flux is a limiting factor for either a neutrino oscillation analysis or for a neutrino cross section measurement. A traditional way to predict the flux is a full chain simulation of the neutrino beamline: from an interaction of primary protons with the target to a neutrino production in meson decays. In this procedure the flux uncertainty would be mainly driven by uncertainties in the production of parent hadrons. A review of recent results of the NA61/SHINE experiment on determination of hadron yields in proton-carbon interactions is presented. The differential cross sections of $\pi^{ \pm}, \mathrm{K}^{ \pm}, \mathrm{p}, \mathrm{K}_{s}^{0}$ and $\Lambda$ provided by NA61/SHINE are presently used in the $\mathrm{T} 2 \mathrm{~K}$ neutrino beam simulation program to reweight hadron yields. They allowed to reduce the flux uncertainty at the neutrino peak energy down to $9 \%$. At the same time, NA61/SHINE results obtained with the T2K replica target, which are used to constrain hadron yields at the surface of the target, will reduce significantly a model dependence of the neutrino beam prediction. Prospects of further improvement of precision of NA61/SHINE measurements in view of the forthcoming runs for energies of the main injector neutrino beamline at FNAL are reviewed.
\end{abstract}

The European Physical Society Conference on High Energy Physics

July 22-29, 2015

Vienna, Austria

\footnotetext{
* Speaker.
} 

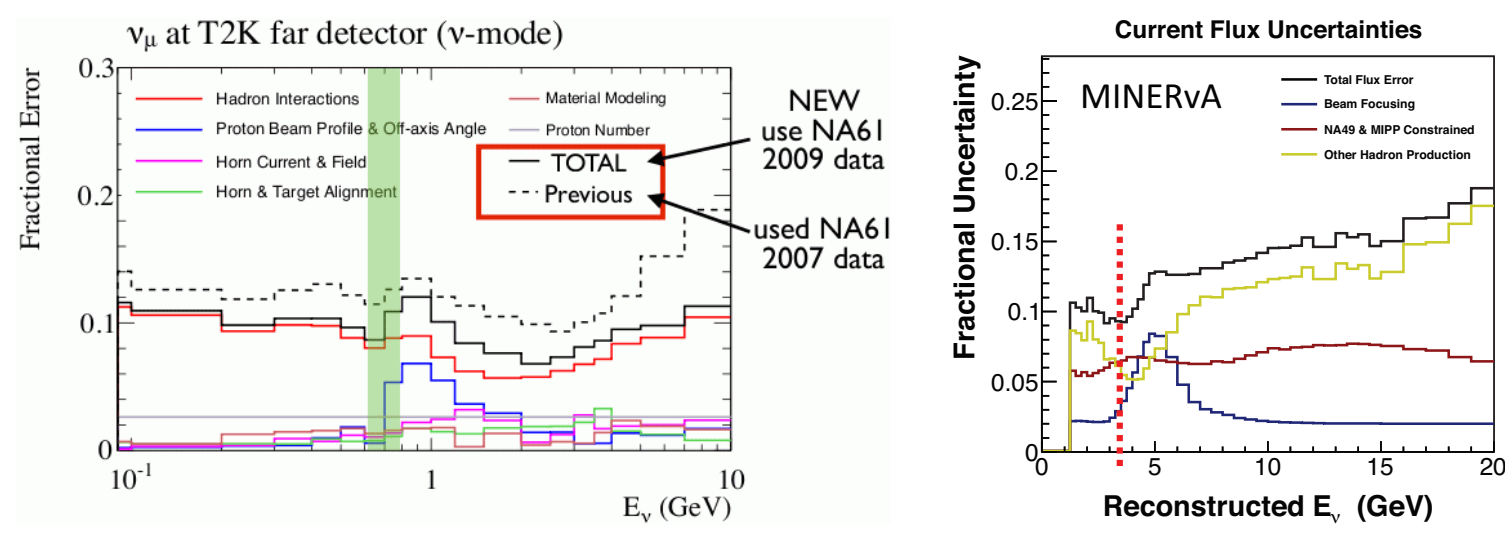

Figure 1: Fractional uncertainty for the $v_{\mu}$ beam calculated for the far detector of T2K (left) and for MINERvA [8] (right) as a function of the $v_{\mu}$ energy, $E_{v}$, are shown. The flux uncertainty of T2K obtained with the NA61/SHINE data taken in 2007 [3] and in 2009 [7] are shown with dashed and solid lines, respectively. Vertical lines at both figures show a peak value of the $E_{v}$ spectrum.

\section{Introduction}

A conventional accelerator neutrino beam is produced in reactions of beam-protons impinging on a long nuclear target to produce hadrons $[1,2]$. The hadrons, including those which are products of reinteractions, are focused by magnetic horns and then travel through a long decay vessel. A part of them decay into neutrinos. Formed in decays of different hadrons at different distances such neutrino beam is neither monochromatic nor pure in flavor. In addition to the main component $v_{\mu}$ (or $\bar{v}_{\mu}$ ) there is a significant admixture of different neutrino flavors $\bar{v}_{\mu}, v_{e}, \bar{v}_{e}\left(\right.$ or $v_{\mu}, v_{e}, \bar{v}_{e}$ ). This admixture is an important source of systematic uncertainty in the neutrino oscillation analysis of LBL experiments.

The neutrino flux prediction requires a full chain simulation of a neutrino beamline: from the primary interaction to the decay with the neutrino production. For each simulated event the interaction chain of hadrons is stored to be re-weighted later with experimentally measured cross sections [3] which, in turn, are obtained in ancillary hadron production experiments [2,4,5]. As a variation of this method the re-weighting of hadron multiplicities could be done at a surface of the target, which would take away a dependence of the results on an interaction model used for simulation inside the target [6].

There are different sources of the systematic uncertainty of the neutrino flux prediction: hadron production, uncertainty in the measurement of the proton beam, the horn current, the target alignment [7]. An example of fractional flux uncertainties estimated for T2K [3] and MINERvA [8] experiments are shown in Fig. 1. A typical value of the absolute flux uncertainty at the neutrino peak energy is $10 \%$. The dominant contribution is coming from uncertainties on the hadron production.

\section{The NA61/SHINE experiment}

The NA61/SHINE apparatus [9] is a large acceptance spectrometer situated at the $\mathrm{H} 2$ beamline of the North Area of CERN SPS. Most of detector components were inherited from the NA49 


\begin{tabular}{|c|c|c|c|c|}
\hline beam & target & year & triggers $\times 10^{6}$ & status of the NA61/SHINE analysis \\
\hline \hline \multirow{4}{*}{$\begin{array}{c}\text { protons } \\
\text { at } 31 \mathrm{GeV} / c\end{array}$} & thin target & 2007 & 0.7 & published: $\pi^{ \pm}[12], K^{+}[13], K_{S}^{0}, \Lambda[14]$ \\
\cline { 2 - 5 } & $2 \mathrm{~cm}\left(0.04 \lambda_{I}\right)$ & 2009 & 5.4 & published: $\pi^{ \pm}, K^{ \pm}, \mathrm{p}, K_{S}^{0}, \Lambda[15]$ \\
\cline { 2 - 5 } & T2K replica & 2007 & 0.2 & published: $\pi^{ \pm}[6]$ \\
\cline { 2 - 5 } & \begin{tabular}{c} 
target \\
\cline { 2 - 5 }
\end{tabular} & 2009 & 2.8 & analysis being finalized [19] \\
\cline { 3 - 5 } & $\left.201.9 \lambda_{I}\right)$ & 2010 & 7.2 & analysis currently on-going \\
\hline
\end{tabular}

Table 1: A summary of the NA61/SHINE data collected for the T2K physics goals [15]. Results of the thin target analysis are used presently in $\mathrm{T} 2 \mathrm{~K}$ [3]. Results of the $\mathrm{T} 2 \mathrm{~K}$ replica target measurements to be integrated to the beamline simulation program of $\mathrm{T} 2 \mathrm{~K}$.

experiment and are described in [10]. The NA61/SHINE spectrometer is built around five TPC detectors. Two of them are placed in the magnetic field produced by superconducting dipoles. Particle identification is performed via measurements of energy losses in TPC $(d E / d x)$ and timeof-flight (ToF) measurements by a scintillator wall installed downstream of the spectrometer. The use of these two sources of a PID information is particularly important in the momentum range of few $\mathrm{GeV} / c$ where the Bethe-Bloch $d E / d x$ bands of charged hadrons cross each other. Thus the $d E / d x$ measurement alone would not be enough to identify particles with sufficient precision and ToF is especially important to resolve this complexity.

One of the physics goals of NA61/SHINE is a measurement of the hadron production for beam energies relevant to LBL neutrino experiments. In particular the T2K experiment primarily relies in the neutrino flux calculation on the hadron measurements performed by NA61/SHINE [3]. The data-taking dedicated to the neutrino main injector beamline (NuMI) in Fermilab has been approved and started in 2015 [11].

For physics runs dedicated to T2K program NA61/SHINE exploited a $31 \mathrm{GeV} / c$ secondary proton beam which interacted with a graphite target. Two target configurations was used. A thin graphite target $\left(0.04 \lambda_{I}\right)$ was aimed to the cross section measurements. The second was a T2K replica target configuration $\left(1.9 \lambda_{I}\right)$ which was aimed to determination of model independent hadron yields outside the target. A summary of the NA61/SHINE data collected for the T2K physics goals is presented in Tab. 1 .

\subsection{Results on cross section measurements}

The first set of data with the $31 \mathrm{GeV} / c$ proton beam and the isotropic graphite target have been collected by NA61/SHINE during the pilot run in 2007. Multiplicities of $\pi^{ \pm}$and $K^{+}$obtained with this data have been published $[12,13]$ and they were used for neutrino flux predictions in T2K up to the end of 2014. In addition to charged hadron spectra NA61/SHINE also published multiplicities of neutral hadrons $K_{S}^{0}$ and $\Lambda$ [14]. However due to low statistics they were not included into the beamline simulation of $\mathrm{T} 2 \mathrm{~K}$.

The high-statistics set of data have been taken by NA61/SHINE in 2009 (see Tab. 1). Analysis of this data have been recently completed and published [15]. The overall precision of measurements have been improved by approximately a factor of 2 except for the lower momentum region where the systematic uncertainty dominates. Furthermore, multiplicities of protons and negatively charged kaons have been published by NA61/SHINE for the first time. Spectra of $\pi^{ \pm}, K^{ \pm}, p, K_{S}^{0}$ 

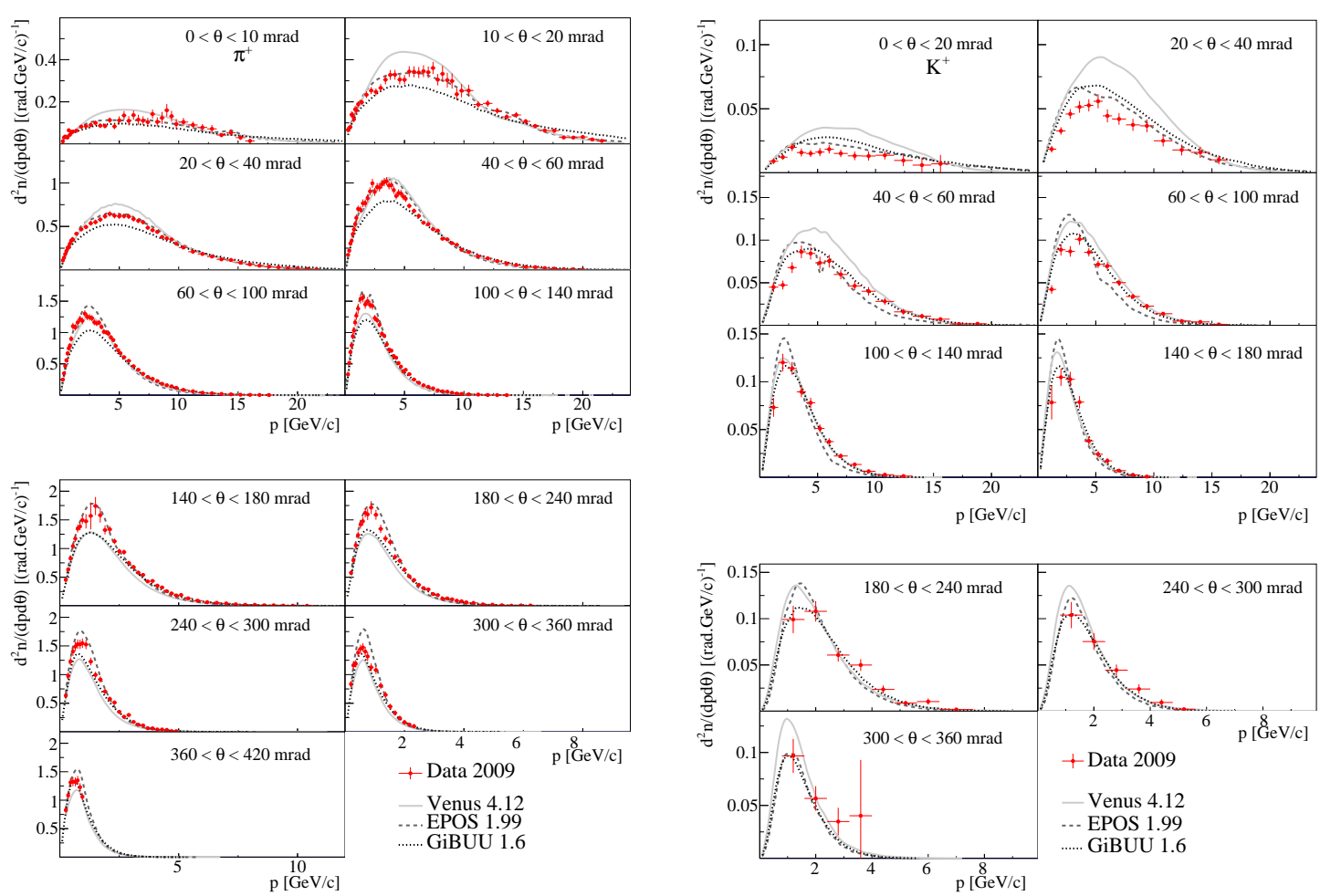

Figure 2: Laboratory momentum distributions of the $\pi^{+}$and $K^{+}$multiplicities produced in p-C interactions at $31 \mathrm{GeV} / c$ in intervals of polar angle, $\theta$, as measured by NA61/SHINE [15]. Graphs are overlapped by various model predictions.

and $\Lambda$ presently being used by $\mathrm{T} 2 \mathrm{~K}$ for the (anti-)neutrino flux predictions [7]. The overall neutrino flux uncertainty obtained with these spectra has been reduced down to $9 \%$ at the $v_{\mu}$ peak energy as shown in Fig. 1. In addition to the use in T2K, the published hadron spectra provide important input to improve hadron production models needed for the interpretation of air showers initiated by ultra high energy cosmic particles.

The measured spectra were compared to predictions of hadron production models: Venus [16], EPOS [17] and GiBUU [18]. As one can see in Fig. 2, none of those models can perfectly describe the spectra.

\subsection{The T2K replica target results}

A significant fraction of intrinsic uncertainties on a predicted neutrino flux in LBL experiments arises from models employed to simulate hadron emission from a long nuclear target. In particular about $30 \%$ of the neutrino flux in T2K arises from particles which are produced in hadronic re-interactions in the target. To constrain the production of those tertiary hadrons in $\mathrm{T} 2 \mathrm{~K}$, one has to use parametrizations (assuming $x_{F}$ scaling) based on NA61/SHINE and other existing hadron production data available in the literature. Another approach proposed by NA61/SHINE is to constrain hadron yields outside a real target. It would provide a model independent estimate of neutrino parents for the $\mathrm{T} 2 \mathrm{~K}$ beamline program. 

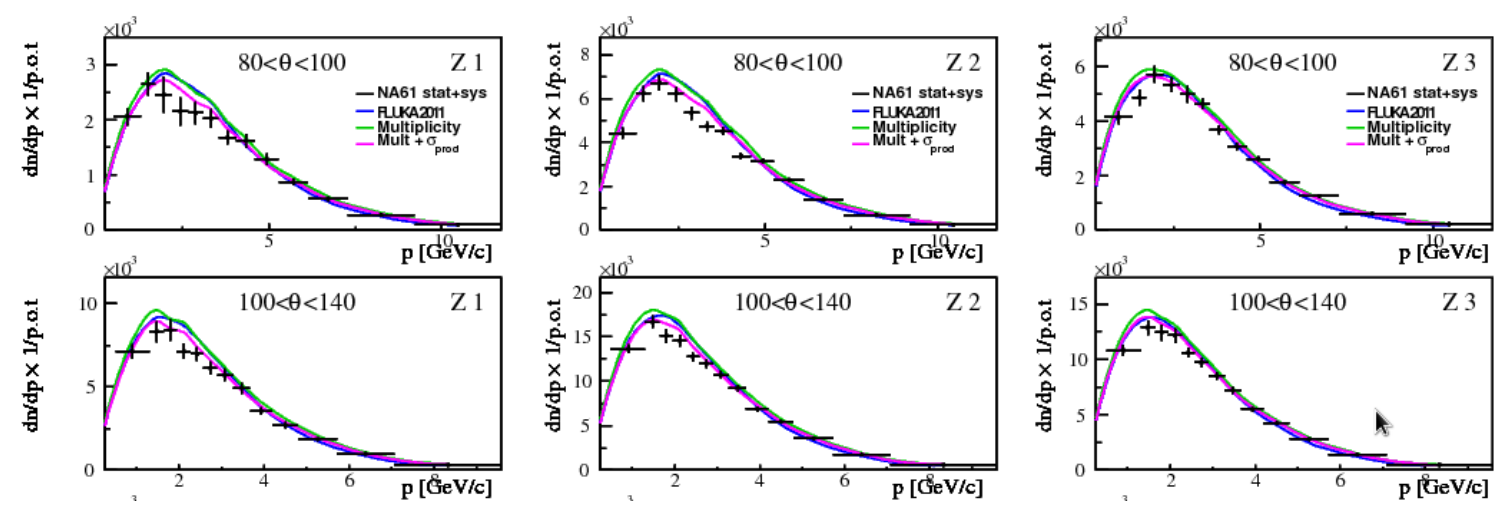

Figure 3: T2K replica target results for positively charged pions with nominal FLUKA predictions (blue), FLUKA re-weighted for the multiplicities (green) and FLUKA re-weighted for multiplicities and production cross section $\sigma_{\text {prod }}$ (magenta) for the three upstream longitudinal bins and in the polar angles between 80 and $140 \mathrm{mrad}$ plotted as a function of momentum [19].

The T2K replica target used in measurements of NA61/SHINE is a $90 \mathrm{~cm}$ long and $2.6 \mathrm{~cm}$ diameter graphite rod. Results of the analysis of the pilot data collected in 2007 have been published [6]. However low statistics did not make them practically useful for T2K. By now the much larger data sample taken in 2009 have been analyzed [19]. The fully-corrected $\pi^{+}$and $\pi^{-}$spectra at the surface of the target are shown in Fig. 3. These spectra have been compared to the prediction calculated with a nominal FLUKA [20] and also with FLUKA re-weighted with experimental measurements. A reasonable agreement is found. The ultimate precision will be obtained using a higher statistics exposure taken in 2010 (see Tab. 1).

\subsection{Comparison of $v$ flux prediction using thin and replica target results of NA61/SHINE}

In order to compare in a consistent way the neutrino flux prediction re-weighted with the thin target procedure [3] and with the T2K replica target results [19], the same settings of the $\mathrm{T} 2 \mathrm{~K}$ beamline program for the flux computation have been used. In case of the replica target computation, only $\pi^{+}$and $\pi^{-}$spectra were set as an input. Other particle species exiting the target surface are re-weighted following the already established thin target procedure. Re-interactions outside of the target (in the focusing horns and along the beam line) are also tuned with the nominal T2K neutrino flux calculation, thus based on the thin target data. The weights have been computed with the NA61/SHINE beam profile which is wider than the one of T2K.

In order to study the different effects of the thin target tuning, the neutrino flux at the far detector of T2K is first re-weighted for the multiplicity only (Fig. 4 left) and then for the multiplicity and production cross section (Fig. 4 right). For the latter case the production cross section was modified by a factor of -0.6 of the quasi-elastic component. It corresponds roughly to lowering the value of the FLUKA production cross section from $241 \mathrm{mb}$ to $221 \mathrm{mb}$. In general results of two flux computations are consistent within uncertainties. However the reduction of the production cross section lead to the better agreement. 

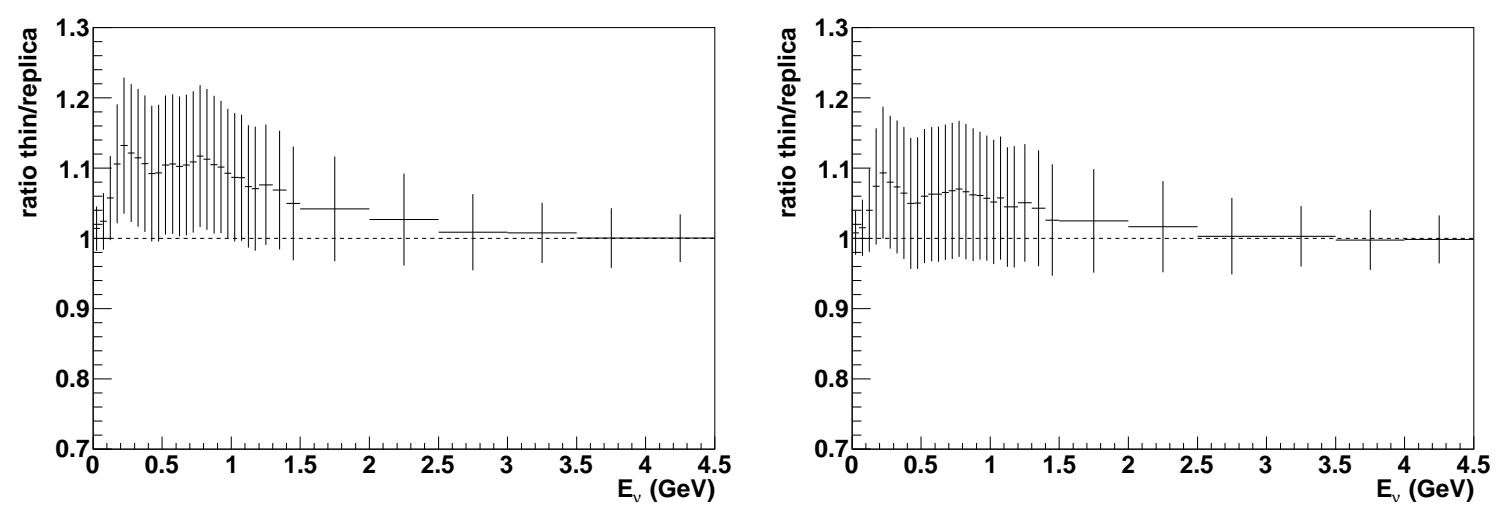

Figure 4: Ratio of the $v_{\mu}$ flux at SK re-weighted with the thin target procedure [3] over the T2K replica target flux [19]. On the left plot the production cross section was not modified for the thin target procedure. On the right the production cross section was rescaled by -0.6 of the quasi-elastic contribution. Vertical error bars show the full uncertainties on the ratio.

\section{Data of NA61/SHINE for the future LBL program}

An extension of the NA61/SHINE physics program for Fermilab neutrino beams was approved by CERN in 2014 [11]. The goal was similar to the T2K program: to collect dedicated hadron production data needed to improve a neutrino beams modeling necessary for ongoing and future experiments at Fermilab. Due to the failure of of the VTX-1 magnet in NA61/SHINE the data taking planned for 2015 was canceled. The run is planned to be resumed in 2016. A data set which will be collected by NA61/SHINE is essential for future neutrino beams in Fermilab including running experiments using the NuMI and LBNF facilities. In particular for the latter one the flux related uncertainty for the expected number of events at the far detector is required to be smaller than $2 \%$ (corresponding value in $\mathrm{T} 2 \mathrm{~K}$ is $3 \%$ ) which will be clearly a challenge [21].

Another future LBL neutrino experiment which can potentially profit from NA61/SHINE measurements is Hyper-Kamiokande [22]. One plans to utilize in the experiment the J-PARC accelerator complex to deliver the neutrino beam to a Kamioka mine. An uncertainty for HyperKamiokande measurements has been estimated using the framework and systematic uncertainties of the presently ongoing T2K experiment. It was shown in Ref. [22] that the flux related uncertainty for the expected number of events at Hyper-K surpasses the next significant contribution by a factor of 2. It emphasizes clearly an importance of precision hadron production measurements which could be provided by NA61/SHINE.

\section{Conclusions}

The accelerator neutrino experiments that measure interaction cross sections and/or perform oscillation analyzes require a precise knowledge of the initial neutrino flux. In the talk two approaches to constrain the neutrino flux using hadron production data have been presented: reweighting hadron cross section at interaction vertices (traditional approach) and re-weighting hadron yields at the target surface (less model dependent, proposed by NA61/SHINE). The application of 
these approaches for the T2K and NuMI beamlines has been discussed. Importance of the hadron production data collected by NA61/SHINE experiment have been emphasized.

\section{References}

[1] S. E. Kopp, Phys.Rept. 439 (2007) 101

[2] M. Bonesini and A.Guglielmi, Phys. Rep. 433 (2006) 65

[3] (T2K collaboration) K. Abe et al., Phys.Rev. D87 (2013) 012001

[4] B. Popov, EPJ Web Conf. 99 (2015) 02002

[5] A. Korzenev, arXiv:1409.7887 [hep-ex], AIP Conf.Proc. 1666 (2015) 150001

[6] (NA61/SHINE collaboration) N. Abgrall et al., Nucl.Instrum.Meth. A701 (2013) 99

[7] L.Zambelli, proceedings of this conference

[8] D.Harris, presented at NuInt14 in May 2014

[9] (NA61/SHINE collaboration) N. Abgrall et al., JINST 9 (2014) P06005

[10] (NA49 collaboration) S.Afanasiev et al., Nucl.Instrum.Meth. A430 (1999) 210

[11] S.R.Johnson et al., CERN-SPSC-2014-032/SPSC-P-330-ADD-7

[12] (NA61/SHINE collaboration) N. Abgrall et al., Phys.Rev. C84 (2011) 034604

[13] (NA61/SHINE collaboration) N. Abgrall et al., Phys.Rev. C85 (2012) 035210

[14] (NA61/SHINE collaboration) N. Abgrall et al., Phys.Rev. C89 (2014) 025205

[15] (NA61/SHINE collaboration) N.Abgrall et al., arXiv:1510.02703 [hep-ex]

[16] (VENUS) K. Werner, Nucl. Phys. A 525 (1991) 501; Phys.Rep. 232 (1993) 87

[17] (EPOS) K. Werner, F.M. Liu, T. Pierog, Phys. Rev. C74 (2006) 044902

[18] (GiBUU) O. Buss et al., Phys. Rept. 512 (2012) 1 ;

K. Gallmeister and U. Mosel, Nucl. Phys. A826 (2009) 151

[19] $\mathrm{PhD}$ thesis of A. Haesler, CERN-THESIS-2015-103

[20] (FLUKA) G. Battistoni et al., AIP Conf. Proc. 896, 31 (2007);

A. Ferrari, P.R. Sala, A. Fasso, J. Ranft, CERN-2005-010, SLAC-R-773, INFN-TC-05-11

T. Böhlen et al., Nuclear Data Sheets 120, 211-214 (2014)

[21] (LBNF collaboration) C. Adams et al., arXiv:1307.7335, FERMILAB-PUB-14-022, 2014

[22] (Hyper-K collaboration) K.Abe et al., arXiv:1502.05199 [hep-ex], PTEP 2015 (2015) 053C02 